\title{
A Musicoterapia e a Música na Medicina como terapia adjuvante no âmbito da Obstetrícia: Uma Revisão Bibliográfica
}

\author{
Edmar Mesquisa Neto , Antonio Marlos Duarte de Melol, Ana Beatriz Callou Sampaio Neves ${ }^{1}$, I

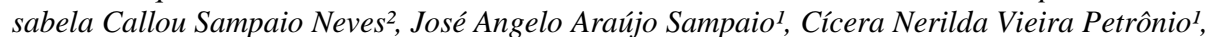 \\ Maria Valéria Leimig Telles ${ }^{1}$, Djailson Ricardo Malheiro ${ }^{1}$.
}

\begin{abstract}
Resumo: A gestação é um dos períodos mais importantes na vida de pai, mãe de filho. Este período é comumente doloroso e difícil, embora seja gratificante e recompensador. No entanto, o tratamento das patologias deste período é difícil, tendo em vista os riscos que os fármacos e as terapias intervencionistas podem causar a saúde dos envolvidos. Tendo isso em vista, esta análise de literatura vista avaliar os progressos recentes da musicoterapia na obstetrícia, esta que vem nos últimos anos sendo estudada em todos os âmbitos da medicina como terapia adjuvante e não seria diferente no processo do nascer. Os trabalhos foram analisados separadamente principalmente conforme o período gestacional na qual tinham abrangido. Optou-se por organizar da seguinte forma: musicoterapia na preconcepção, no pré-natal, no parto normal, no parto cesáreo, musicoterapia sobre o pai e por fim analisamos de uma forma geral a metodologia dos ensaios clínicos mais recentes.
\end{abstract}

Palavras chave: musicoterapia, obstetrícia, saúde.

\section{Music Therapy and Music in Medicine as Auxiliary Therapy in Obstetrics: A Bibliographic Review}

\begin{abstract}
Gestation is one of the most important periods in the life of father, mother of child. This period is often painful and difficult, although rewarding and gratifying. However, the treatment of the pathologies of this period is difficult, given the risks that drugs and interventional therapies can cause to the health of those involved. With this in mind, this literature review evaluates the recent advances in music therapy in obstetrics, which has been studied in recent years in all areas of medicine as adjuvant therapy and would not be different in the process of birth. The studies were analyzed separately mainly according to the gestational period in which they had covered. It was decided to organize as follows: music therapy in preconception, prenatal, normal delivery, cesarean delivery, music therapy on the father and finally we analyze the methodology of the most recent clinical trials in general.
\end{abstract}

Keywords: music therapy, obstetrics, health.

\section{Introdução}

O ser humano é um ser que vive em equilíbrio, podendo esse ser rompido pelas mais diversas patologias, sejam elas de origem física ou emocional. Essas desordens tem a capacidade de romper ciclos fisiológicos e suas minuciosas reações químicas que ocorrem

\footnotetext{
${ }^{1}$ Faculdade de Medicina Estácio de Juazeiro do Norte, Juazeiro do Norte, Ceará, Brasil.

2 Prefeitura Municipal de Juazeiro do Norte, Juazeiro do Norte, Ceará, Brasil. Contato: valerialeimigtelles@ hotmail.com Autor correspondente: Edmar Mesquisa Neto - Curso de Medicina - Estacio-FMJ . Rua Tenente Raimundo Rocha, S/N, Planalto, Juazeiro do Norte, Ceará, Brasil, CEP 63.048-080. E-mail: emesneto@gmail.com.
} 
dentro de todos os indivíduos em cada momento de sua vida.

Tendo em vista a manutenção da homeostase do ser humano, várias artes têm sido estudadas a fim de avaliar seu potencial relaxante e seus efeitos na fisiologia do indivíduo. A música, como uma das mais antigas formas de expressão do indivíduo, tem sido um alvo constante de estudos médicos recentes no que diz respeito aos seus efeitos nos mais diversos grupos de pacientes. Existem dados bem documentados quanto a sua importância na melhora da concentração, atenção e resistência física de pacientes de grupos gerais (WULFF et al, 2017).

Não seria diferente no caso específico das gestantes. Esse grupo é especialmente acometido por estresse indolente durante todo o período da gestação, parto e pós-parto. O medo de que algo não ocorra conforme o planejamento ideal e que a recuperação de alguma intempérie seja lenta é uma constante. Para estes, a música tem se mostrado benéfica nos campos emocionais, intelectuais, psicológicos, fisiológicos e sociais (GONZALES et al, 2017), além de ter efeitos benéficos específicos sobre a depressão e dor pós-parto normal, ansiedade e uma maior satisfação geral no período que sucede ao nascimento. Sendo assim, a musicoterapia torna-se uma alternativa importante de medicina complementar não farmacológica de fácil realização prática, com baixo custo, e efeitos imediatos para este grupo (SIMAVLI et al, 2014).

Os efeitos da ansiedade materna, não se limitam somente a mãe. O feto, é parte da homeostase de sua genitora e é diretamente influenciado pelo seu estado. É sabido que problemas como a ansiedade durante a gestação podem ter efeitos diretos no desenvolvimento do bebê, impactando inclusive em parâmetros como distúrbios hemodinâmicos, estatura, peso e número elevado de partos cesáreos (BETEGÓN et al, 2017). Esses números podem ser positivamente alterados quando as pacientes gestantes são submetidas as sessões de musicoterapia de forma regular e bem estabelecida (GARCIA-GONZALEZ et al, 2018).

Esses efeitos podem ser explicados devido a uma série de mecanismos fisiológicos que são ativados ou debelados no momento da escuta da música ou mesmo a longo prazo. Um dos principais neurotransmissores relacionados a musicoterapia é a serotonina natural que cria um estado de relaxamento, já a acetilcolina que também tem sua liberação potencializada, tem efeito na redução da frequência cardíaca, redução da pressão arterial e aumento do fluxo sanguíneo para órgãos nobres (BETEGÓN et al, 2017). Não obstante, os glicocorticoides como o cortisol, que é fortemente relacionado ao estado de estresse, têm sua liberação reduzida, com consequente benefício no desenvolvimento do bebê, já que esse é capaz de atravessar a barreira placentária e interferir diretamente na fisiologia fetal (GARCIA-GONZALEZ et al, 2018). 
Foram realizados também, estudos para tratamentos objetivos. No caso da infertilidade tratada com fertilização in vitro, a musicoterapia foi utilizada como terapia adjuvante já que é documentada a relação de níveis elevados de ansiedade e infertilidade. $\mathrm{O}$ estudo mostrou que o nível de ansiedade foi significativamente reduzido, porém sem impacto na infertilidade em relação ao grupo-controle. Como resolução a essa dúvida, foi sugerida a realização de estudos com amostragens maiores (ABA et al, 2017).

A perspectiva paterna também foi abordada, em estudo foi avaliado o nível de ansiedade paterna de pais de primeira vez e o impacto da musicoterapia nestes indivíduos devido ao conhecimento de que a ansiedade paterna é capaz de influenciar na relação mãe-bebê além de impactar na recuperação e saúde dos pares. Foi identificado após aplicação de testes específicos em escala visual, que os pais submetidos a musicoterapia tinham uma visão global do parto e uma satisfação geral melhoradas após a musicoterapia, além de terem melhores níveis de ansiedade geral (LABRAGUE; MCENROE-PETITTE, 2014).

Esse estudo, visa avaliar a musicoterapia como terapia complementar na obstetrícia e avaliar seus impactos na redução de ansiedade e melhoria da experiência geral da gestante, pai e bebê do momento da anticoncepção ao momento do delivramento, seja ele espontâneo, induzido ou realizado por via cirúrgica. Analisando a musicoterapia como única terapia adjuvante com objetivo de melhorar os parâmetros relacionados a obstetrícia.

\section{Metodologia}

\section{Delineamento do estudo}

O trabalho consistiu numa revisão bibliográfica sobre a musicoterapia utilizada no contexto da obstetrícia, produzida a partir de uma pesquisa de literatura com o intuito de atingir os objetivos propostos.

\section{Coleta de dados}

A pesquisa ocorreu no período de 12 de fevereiro de 2018 a 12 de maio de 2018. Os dados foram coletados através de uma pesquisa à plataforma pubmed com as seguintes palavras 
chaves de forma separada ou com combinações: Music, musctherapy, obstetric, pain, delivery, anxiety, maternal, fetal, labour, father, sleep.

Foi feita uma pesquisa rápida com o intuito de selecionar artigos que tenham relevância para o estudo proposto. Assim, foram encontradas 34 referências, de acervo virtual, delimitando o tempo de pesquisa dos anos de 2010 a 2018, com o intuito de verificar os avanços recentes da abordagem ao tema. Seis artigos foram excluídos por não atenderem os critérios abaixo descritos.

\section{Critérios de inclusão e exclusão}

Durante a pesquisa, foram selecionados artigos que tinham relevância com o tema proposto, dando prioridade aos artigos mais recentes e que fossem estudos de caso, revisões sistemáticas e bibliográficas. Sendo estes realizados por equipes multiprofissionais que utilizassem como método de terapia adjuvante a musicoterapia no ambiente específico da obstetrícia, seja ela aplicada a mãe, ao feto ou aos componentes das famílias impactados pelo processo de nascimento, seja ele durante o ato do delivramento em si ou até mesmo na anticoncepção.

Optaram-se por excluir estudos que não tivessem metodologia satisfatória, que não utilizassem a musicoterapia de forma adjuvante no estudo em questão ou que não fossem escritos em inglês.

\section{Aspectos éticos}

Os dados utilizados para a confecção desse trabalho foram obtidos através de uma revisão integral de estudos previamente elaborados, realizando uma análise e interpretação dos mesmos de modo a respeitar o sentido original transmitido pelos autores devidamente citados.

Por tratar-se de uma revisão de literatura não houve necessidade de aprovação pelo Comitê de Ética e Pesquisa (CEP). O estudo obedeceu aos aspectos éticos da pesquisa conforme a resolução 466/12 do Conselho Nacional de Saúde (CNS). 


\section{Revisão da Literatura}

\section{Conceito de musicoterapia e música na medicina}

A música é uma das mais antigas artes da história da humanidade, sendo seus achados tão antigos quanto o próprio homem. Instrumentos rudimentares, foram encontrados ainda com o homem de Neandertal. Sendo assim, suas aplicabilidades nos mais diversos casos foram testadas e estudadas, não sendo diferente dentro da prática médica (CONRAD, 2010).

Desde a história antiga e durante a antiguidade clássica, existem achados importantes do apoio da música ou até sua exclusividade no tratamento das patologias. As civilizações mesopotâmicas como os assírios, as do norte do oriente médio como os hebreus, as que margeavam o rio Nilo como os Egípcios tinham a música como adjuvante nos mais diversos tratamentos, já as civilizações da antiguidade clássica como a Grécia, já começaram a teorizar a importância da música neste contexto, tendo expoentes importantes como Platão e Aristóteles discorrendo sobre o assunto (THAUT, 2015).

A música como ferramenta de alivio a ansiedade e de cura de doenças é algo instintivo ao ser humano, hábito este que pode ser notado quando vemos atos simples como uma mãe que cantarola ao seu bebê para que se acalme, ou uma tribo indígena antiga que usa instrumentos musicais de percussão e cantos bem ensaiados para rituais de cura de patologias (RAGHAVAN; EKNOYAN, 2013). Dessa maneira, como sendo algo tão intrínseco ao indivíduo, a música carece de ser estudada cuidadosamente em todas as suas aplicações e possibilidades.

Tendo em vista isso, se faz extremamente importante definir os conceitos de musicoterapia e música na medicina a fim de aprimorar o uso de ambas as práticas. O conceito é baseado primariamente no tipo de profissional que realiza a prática música dentro do âmbito da saúde.

O conceito de musicoterapia se restringe ao profissional musicoterapeuta, que faz uso da música e que tem treinamento específico para o uso da prática. Já o termo música em medicina é bem mais amplo, podendo a prática ser estendida para todos os tipos de profissionais da saúde, seja médico, enfermeiro, fisioterapeuta e etc, sendo ele tendo treinamento especifico ou não (YINGER; GOODING, 2015).

É notório que nos estudos aplicados há uma predominância da realização de estudos conduzidos por profissionais não musicoterapeutas, sendo assim a maioria dos estudos, sejam 
eles ensaios clínicos ou análises de literatura são baseados na música na medicina e não na musicoterapia propriamente dita (CHANDA, LEVITIN, 2013). Do ponto de vista técnico essa predominância pode ser prejudicial, já que os profissionais não têm treinamento específico sobre o assunto na qual realizam os estudos, podendo empobrecer o material disponível sendo necessário mais estudos comparando os diferentes tipos de intervenção (YINGER; GOODING, 2015).

\section{Musicoterapia na preconcepção}

Existem poucos estudos recentes avaliando os possíveis efeitos da musicoterapia no que diz respeito a fertilidade e redução de patologias relacionadas a concepção. Os estudos encontrados se referem aos efeitos da musicoterapia na fertilização in vitro, não sendo encontrados estudos referentes a fertilização assistida ou desassistida ou no que diga respeito as patologias de implantação ou defeitos de segmentação embriológica.

O estudo realizado por (MURPHY et al, 2014) foi um ensaio randomizado em que se foi utilizada a musicoterapia com o objetivo de se avaliar a redução da ansiedade e os possíveis efeitos benéficos no aumento da fertilidade de 181 mulheres durante a fertilização in vitro. Neste estudo, foi utilizada a harpa como instrumento melódio e relaxante por 20 minutos sob supervisão de um musicoterapeuta certificado. Não foi especificada a taxa de batimentos por minuto na qual a música era conduzida, e o volume que era utilizado durante as músicas. A terapia era realizada durante a transferência de embriões e as pacientes foram submetidos a testes e avaliação do nível total de estresse.

Os pacientes tinham suas pressões arteriais, frequências cardíaca e respiratória monitoradas e respondiam a um questionário que avaliava os níveis de ansiedade linear e de estado antes e depois do ensaio clínico (MURPHY et al, 2014).

Como resultado, não foram encontradas diferenças significativas entre as frequências cardíaca e respiratória nem houve uma diferença significativa dos níveis de ansiedade do início do estudo, porém houve significativa redução dos níveis de ansiedade pós fertilização. Como conclusão, observou-se uma taxa de fertilidade de 53\% nas mulheres submetidas a musicoterapia e de $48 \%$ em relação ao grupo controle (MURPHY et al, 2014). 
Esse estudo, de forma isolada tem um resultado questionável tendo em vista a pouca diferença entre os grupos controle e de intervenção. Isso se dá também devido ao fato de que poucos dados objetivos tiveram qualquer mudança positiva. A pouca especificação de como era realizada a musicoterapia, é um dos pontos fracos do estudo.

O estudo realizado por (ABA et al, 2017) foi também um ensaio randomizado realizado com 186 mulheres inférteis no hospital americano na Turquia que eram submetidos a fertilização in vitro. Para esse estudo, foi realizada musicoterapia por vinte e oito minutos, uma hora antes e após o procedimento de fertilização. Um musicoterapeuta treinado decidiu por reproduzir concertos e violino e viola clássica de Bach, Martini e Mozart num volume de 45 $\mathrm{Db}$, porém não foi especificado o andamento médio das músicas aplicadas, sendo caracterizadas apenas por possuir um efeito calmante.

Os pacientes eram submetidos ao preenchimento de um formulário com informações pessoais e um teste que avalia os níveis de ansiedade linear e de estado antes e após a realização das terapias (ABA et al, 2017).

Os resultados apontaram uma significante redução da quantidade de ansiedade linear e estado após a fertilização in vitro, no entanto, não apontaram diferenças estatísticas significativas nos níveis de ansiedade antes do procedimento (ABA et al, 2017). Isto por si só pode apontar para a necessidade de mais de um ciclo de musicoterapia para que haja resultado real ou aponte a ineficácia da musicoterapia antes da fertilização.

Não houveram diferenças significativas entre os níveis de fertilidade entre o grupo controle e o grupo submetido a terapia, neste estudo, $48,3 \%$ das mulheres do grupo de musicoterapia e $46,4 \%$ das mulheres do grupo controle foi fertilizado. $\mathrm{O}$ estudo sugeriu a realização de novos ensaios randomizados a fim de avaliar a possível eficácia dos métodos para a fertilidade (ABA et al, 2017).

Ambos os estudos apresentaram resultados pouco positivos em relação a fertilidade da mulher submetida a musicoterapia, no entanto, sugeriram uma importante redução dos níveis de ansiedade linear-estado, no período pós fertilização. Devido à escassez de estudos que atendem ao propósito da fertilização, os dados são pouco conclusivos, principalmente devido a uma diferença discreta entre os escores que dizem respeito a fertilização, mesmo os estudos se tratando de ensaios clínicos randomizados. Se faz necessária a realização de mais ensaios clínicos a fim de que seja feita a melhor elucidação das dúvidas propostas pelos estudos. 


\section{Musicoterapia no pré-natal}

O período de pré-natal é vital e determinante para o sucesso da gestação e do processo de nascimento. Com um bom acompanhamento, torna-se possível prever, diagnosticar, acompanhar a evolução e até mesmo tratar um sem número de patologias que afetariam a vida do concepto e da mãe. Isso mostra-se ainda mais relevante tendo em vista que existem evidências que transtornos como depressão e ansiedade na vida adulta pode ser prevista a partir do histórico obstétrico e pré-natal da gestante (ESSAU et al, 2018).

Neste contexto, foram realizados trabalhos afim de avaliar o impacto da terapia com música neste período e avaliados seus efeitos durante a aplicação e após o nascimento do bebê. Sendo possível observar o maior número de trabalhos disponíveis na plataforma no contexto deste estudo.

Uma pesquisa realizada por (NWEBUBE; GLOVER; STEWART, 2017) realizou um estudo randomizado com duzentas e vinte e duas mulheres grávidas e voluntárias, com mais de 18 anos e que falavam língua inglesa. Foram alocados cento e onze mulheres para o grupo controle e cento e onze mulheres para o grupo que sofreria a intervenção. As pacientes foram solicitadas a preencher um questionário de ansiedade estado e linear e a escala Edimburgo de depressão pós-natal e foram submetidas a vinte minutos diários de musicoterapia durante 12 semanas, após este período preencheram as escalas novamente.

Os examinadores deste estudo, estregaram música especialmente composta para o prénatal em formato de MP3. A música foi descrita como tendo tempos específicos, ritmos e frases destinadas a fins de efeitos calmantes, no entanto dados tempos e ritmos não foram especificados. Elementos de canções de ninar, ralentificação musical e repetições foram utilizadas como elementos calmantes nas composições originais, além das letras possuírem elementos relevantes para mulheres gravidas (NWEBUBE; GLOVER; STEWART, 2017).

Não foi orientado quanto ao volume da execução das músicas devido ao fato de que a música era reproduzida em casa, sem controle de profissional no momento da escuta. Ao fim do estudo foi verificada redução significativa dos níveis de ansiedade e depressão no grupo musical, enquanto não houveram diferenças significativas no grupo controle (NWEBUBE; GLOVER; STEWART, 2017).

No entanto, vale ressaltar que os níveis de ansiedade e depressão inicial nos dois grupos estava acima da média nacional para gestantes, o eu pode significar o viés de que mulheres 
grávidas sabidamente com depressão e ansiedade se voluntariaram para o estudo (NWEBUBE; GLOVER; STEWART, 2017).

No estudo randomizado realizado por (GARCIA-GONZALEZ et al, 2018), foi aplicada a musicoterapia a 409 gestantes submetidas a pré-natal de rotina, que possuíam mais de 18 anos, estavam no terceiro trimestre de gestação, eram primíparas, não utilizavam nenhum tipo de droga além das prescritas pelo ginecologista, não tinham diagnóstico de diabetes mellitus gestacional ou pré-eclâmpsia e que possuíam fetos únicos, vivos sem anormalidades detectadas.

Ambos os grupos eram submetidos a uma cardiotocografia durante uma vibroacusticoestimulação. Foram submetidos também a um questionário a fim de avaliar os níveis de ansiedade linear e estado, sendo que o grupo controle foi submetido no terceiro trimestre de gestação e o grupo de intervenção era submetido aos testes antes e após as sessões de musicoterapia (GARCIA-GONZALEZ et al, 2018).

As gestantes do grupo de intervenção foram submetidas a 14 sessões de musicoterapia, realizadas entre 20 a 40 minutos por sessão, três vezes por semana no mesmo horário preferencialmente. As músicas selecionadas para o estudo tinham menos de 60-75 batimentos por minuto, mimetizando a frequência cardíaca ideal. O volume variou de 60-70 Db. Foi oferecido um $\mathrm{Cd}$ com temática composta para gestante, com músicas instrumentais de instrumentos acústicos (GARCIA-GONZALEZ et al, 2018).

Após realizar-se o estudo, ficou evidenciado que houve redução significativa da ansiedade linear e de estado nas mulheres participantes do grupo de intervenção musical durante o terceiro trimestre de gestação (GARCIA-GONZALEZ et al, 2018).

Todavia, a cardiotocografia se demonstrou como sendo ansiogênica para as gestantes. Esse fato torna esse estudo especialmente importante, tendo em vista que a cardiotocografia é um e exame comumente utilizado na prática clínica e é um dos melhores preditores da vitalidade fetal (GARCIA-GONZALEZ et al, 2018). Esse ponto levanta uma dificuldade para esse tipo de pesquisa, tendo em vista que estudos como estes requerem que a paciente não tenha agravos em seus níveis de ansiedade, necessitando-se avaliar o basal.

O estudo realizado por (GEBUZA et al, 2017) foi conduzido com 55 mulheres gestantes do terceiro trimestre da gestação, com um média de 36 semanas gestacionais, que não tinham risco de parto pré-maturo e sem dinâmica uterina presente. 
A metodologia consistia concisamente na realização de uma cardiotocografia durante 30 minutos seguida por uma sessão de 15 minutos de musicoterapia onde foram realizados registros cardiotocográficos durante e após a estimulação musical (GEBUZA et al, 2017).

As gestantes ouviam música clássica, com uma rítmica semelhante a uma polca, composta por Mozart e Strauss, com andamento de 160-175 BPM, com volume de 65 Db a partir dos alto-falantes de um laptop (GEBUZA et al, 2017).

Esse ponto em especial, torna este estudo especialmente interessante, já que a literatura consensualmente utiliza taxas de BPM entre 60 e 80 e rítmicas descritas como calmas, o trabalho testa justamente um andamento muito acelerado e uma rítmica conhecida como sendo contagiante.

O ensaio clínico demonstrou um aumento dos movimentos fetais durante a exposição a música, durante a cardiotocografia. Sendo que a variabilidade cardíaca girou em torno de 10 a 15 batimentos por minuto, o que é benéfico ao bem-estar do fato (GEBUZA et al, 2017).

Outro estudo, realizado por (CHANG et al, 2015), utilizou uma amostra de 296 mulheres grávidas com mais de 18 anos de idade, idade gestacional de 17 semanas, expectativa de parto vaginal sem complicações, sem morte fetal ou natimorto anterior e de língua chinesa.

Para a avaliação das gestantes, foi utilizado a escala de classificação de estresse gestacional, escala de estresse estado e escala de fixação materno-fetal. As mulheres eram orientadas a ouvir músicas entre os estilos de Crystal music, música clássica, sons da natureza, e canções de ninar, na qual tinham BPM's variados e não havia restrição de volume da escuta (CHANG et al, 2015).

As gestantes podiam ouvir a música durante o repouso, laser ou enquanto desempenhavam suas atividades cotidianas. As sessões eram diárias e o estudo demandou 14 sessões de musicoterapia (CHANG et al, 2015).

O ensaio clínico, durante a análise de dados, demonstrou uma redução significativa dos níveis tensão, porém não demonstrou uma redução da percepção geral do estresse o que não é percebido em outros tipos de ensaios (CHANG et al, 2015). Essa situação pode ter ocorrido devido ao fato de que essas sessões não tinham horário nem lugar próprio para ocorrer, além de não serem supervisionadas. Isso é viés bastante forte e difícil de se avaliar em um estudo como este.

Durante uma meta-análise realizada por (VAN WILLENSWAARD, 2017), ficou evidenciado que não existe redução significativa no estresse geral ou no estresse percebido 
durante a gestação, no entanto, é possível inferir que existe importante redução dos níveis de estresse logo após a intervenção musical.

Ficou evidenciado também que os níveis de ansiedade e estresse variam de acordo com a idade gestacional do bebê, portanto os efeitos da musicoterapia podem variar igualmente de acordo com isto (VAN WILLENSWAARD, 2017).

Outro ensaio clínico randomizado foi realizado por (LIU et al, 2015) e tinha o intuito de avaliar a qualidade do sono de mulheres grávidas, maiores de 18 anos, que estavam entre 18 e 34 semanas de gestação e tinham um índice de Pittsburgh maior que 5, o que indica uma qualidade ruim de sono. Cento e vinte e uma mulheres foram selecionadas para o estudo e divididas entre grupo controle e de intervenção.

Esse estudo foge do convencional dos demais estudos avaliando não a ansiedade e o estresse, mas outro ponto importante do bem-estar materno. A gestação pode alterar consideravelmente a qualidade do sono. Mudanças fisiológicas como ganho de peso e edema de vias aéreas superiores podem predispor a distúrbios de sono, estes que se tornam cada vez mais prevalentes conforme a gravidez progride (PAMIDI; KIMOFF, 2017).

Foi ofertada as gestantes, cinco CDs de música com 30 minutos cada e foi orientado que se escutasse o CD logo antes de dormir. Os CDs continham música clássica das mais variadas origens, soavam a um BPM de 60-80, imitando o pulso humano. Não houveram orientações quanto ao volume na qual a música deveria ser executada (LIU et al, 2015).

Foram aplicados testes de avaliação dos níveis de ansiedade, depressão e qualidade do sono e os mesmos foram repetido após duas semanas de terapia diária. Quando aplicados questionários, foi descoberto uma redução nos níveis de ansiedade e depressão e uma melhora da qualidade do sono das gestantes (LIU et al, 2015).

O ensaio clínico realizado por (TOKER; KÖMÜRCÜ, 2017) tinha como objetivo estudar os efeitos da musicoterapia na pré-eclâmpsia. Para tal, 70 mulheres com o diagnóstico da patologia foram recrutadas para o estudo. As mulheres estavam internadas a mais de 5 dias, estavam com mais de 30 semanas de gestação. A avaliação se deu por meio de uma cardiotocografia e a música era aplicada somente pelo musicoterapeuta, enquanto as pacientes estavam hospitalizadas.

As gestantes foram tratadas com sessões diárias de musicoterapia, onde era reproduzida música clássica turca durante 30 minutos por sete dias através de um MP3 player enquanto 
estivessem de repouso e deitadas. O grupo controle também era orientado quando a 30 minutos de repouso, porém não ouviam música (TOKER; KÖMÜRCÜ, 2017).

Foram realizados os testes cardiotocográficos antes e após os 30 minutos de sessões. Dados como pressão arterial, frequência cardíaca e respiratória da mãe também eram coletados antes e após as sessões de musicoterapia (TOKER; KÖMÜRCÜ, 2017).

Durante a análise de dados, ficou evidenciado que as gestantes tiveram um efeito mínimo nos seus níveis de ansiedade, pressão arterial sistêmica, frequência cardíaca e respiratória (TOKER; KÖMÜRCÜ, 2017). Fato este, entra em desacordo com todos os estudos pesquisados até então neste trabalho.

Os dados tornaram-se significativos quando em comparação com o grupo controle. Isso se torna ainda mais verdadeiro quando se é avaliado o nível de pressão arterial isoladamente, esta que apresentou os níveis de redução mais importantes (TOKER; KÖMÜRCÜ, 2017).

Explicando esse fato, Toker e Kömürcü (2017) argumentaram quanto a crescente tensão que se aplica sobre a mãe que sofre de pré-eclâmpsia durante os dias de internação. A pouco variação dos dados analisados, por si só se mostram benéficos, tendo em vista que o comum para estes casos é que as implicações fisiológicas e emocionais sejam maléficas em uma internação a longo prazo.

Como conclusão, Toker e Kömürcü (2017) sugeriram que fossem feitos estudos com o intuito específico de avaliar a redução da pressão arterial sistêmica em pacientes com préeclâmpsia, o que é bastante válido, levando em consideração que a principal alteração fisiológica da pré-eclâmpsia, seja ela leve ou grave é o aumento pressórico da gestante a partir das 22 semanas de gestação.

Quando fazemos a análise desses trabalhos em conjunto, fica evidente a importância de terapias adjuvantes neste período de intensa preparação para o parto. São meses onde a ansiedade, depressão e estresse são crescentes e determinantes para que haja uma satisfação clínica no que diz respeito ao trabalho de parto e no decorrer de toda a parentalidade.

No entanto, a pouca uniformidade e padronização dos modelos de estudo torna-se prejudicial. Outro ponto relevante neste caso é o excesso de trabalhos que trabalham o tema da ansiedade, estresse e depressão em detrimento de outros pontos relevantes, como a variação de pressão arterial sistêmica, qualidade de sono, frequência cardíaca, inter-relação com o companheiro entre outros pontos que podem ser abordados. 


\section{Musicoterapia no parto normal}

O momento do parto é um marco importante na vida de uma mulher, sendo o início de uma relação mais próxima entre uma mãe e um bebê. No entanto, sendo este um parto normal, torna-se também uma das experiências mais dolorosas da vida de uma mulher. Terapias não medicamentosas e relaxantes podem atenuar o sofrimento nesse momento (WULFF et al, 2017). Sendo assim, a música como terapia adjuvante, pode vir a melhorar a percepção geral da mãe quanto a estes aspectos.

Tendo em vista isso, (DEHCHESHMEH, RAFIEI, 2015), realizam um estudo prospectivo e randomizado com 90 mulheres entre a 38 e 40 semanas gestacionais, primíparas, com apresentação cefálica e saúde mental e físicas em bom estado que deram entrada no hospital em trabalho de parto.

Dehcheshmeh e Rafiei (2015), dividiram aleatoriamente as gestantes em três grupos distintos e os nomearam em grupo "A", "B" ou "C". O grupo "A" recebeu musicoterapia durante 30 minutos. Essas mulheres recebiam um player com músicas executadas por um piano. O trabalho não especificou o ritmo musical, a taxa de BPM da música especificada e o controle de volume dos aparelhos. O grupo "B" era submetido a sessões de massagem com gelo nos pontos Hoku, em uma posição de escolha das gestantes e por um profissional treinado. Já ao grupo "C" foram oferecidos os cuidados usuais da maternidade.

As pacientes, então, preenchiam uma escala visual analógica (EVA), graduada de 0 a 10, a partir da fase ativa do trabalho de parto (4 cm de dilatação) e a partir daí aos 6 e aos $8 \mathrm{~cm}$ de dilatação (DEHCHESHMEH, RAFIEI, 2015).

Com o final do trabalho, pode-se perceber que a música tinha efeito relaxante semelhante as massagens (DEHCHESHMEH, RAFIEI, 2015). No entanto, esse tipo de estudo comparativo torna-se limitado, tendo em visto a escassez de estudos neste sentido e a necessidade neste momento de estabelecer um comparativo da musicoterapia entre suas próprias nuances.

O artigo original realizado por (TABARRO et al, 2010), foi um ensaio clinico dirigido por enfermeiros do interior de São Paulo e incluiu gestantes que faziam pré-natal de baixo risco na atenção primária de saúde, fizeram cinco sessões de sensibilização musical, que se comprometeram a dar à luz nas maternidades cadastradas para o trabalho e que tiveram seu parto no período de termo. 
Nas sessões de sensibilização musical, as gestantes se acomodavam em salas reservadas, deitavam-se com colchonetes e ouviam de oito a dez músicas previamente selecionadas para cada sessão e com melodias baseadas na música clássica, romântica e do folclore japonês, num período de trinta e cinco a quarenta e cinco minutos (TABARRO et al, 2010).

Ao fim das sessões, as gestantes anotavam quais músicas trouxeram lembranças e sensações boas ou ruins. Essas anotações foram levadas em consideração para a confecção de um CD individualizado para cada gestante, que seria tocado no momento do trabalho de parto (TABARRO et al, 2010).

O acompanhamento e coleta de dados se baseou na ficha de evolução da gestante e em uma entrevista semiestruturada realizada nas primeiras 24 horas após o parto, entre 2 e 4 meses após o parto e no terceiro mês após o parto, este se referindo a percepção da musicoterapia sobre o bebê (TABARRO et al, 2010).

O primeiro problema grave desta pesquisa foi a amostra final do trabalho. Apenas 12 gestantes preencheram todos os critérios necessários para que seja concluída sua percepção da musicoterapia. Já no que diz respeito a musicoterapia referente ao bebê, 20 mães preencheram os critérios estipulados, o que ainda é uma amostra pequena.

Outro problema visível deste trabalho foi a ausência de um grupo controle para que se pudesse estabelecer um comparativo, o que levanta dúvidas quanto a relevância da amostra. Esse aspecto tentou ser atenuado trazendo ao estudo momentos, durante o trabalho de parto, na qual a música era retirada para que a própria mãe pudesse relatar a diferença entre as sensações. No entanto, isso torna-se impreciso, tendo em vista que conforme diversos estudos já apresentados neste mesmo trabalho, a musicoterapia tem efeitos também a médio e longo prazo.

Um ponto alto do trabalho foi a abordagem do desejo musical pessoal e o fato de apresentar as músicas oferecidas e as mais escolhidas pelas gestantes. É possível deduzir empiricamente que as músicas mais escolhidas, são as que geram melhores sensações a gestante.

Tabarro et al (2010) conclui inferindo a partir dos relatos das próprias gestantes que o trabalho de parto assistido de musicoterapia teve forte impacto positivo sobre as gestantes, conforme seu próprio relato. Fica claro que os momentos em que a música estiveram presentes levaram mais segurança e conforto para em gestante quando em comparação aos momentos de pausa. 
O trabalho realizado por (SMAVLI, 2014) reuniu 156 mulheres primíparas com mais de 37 semanas de gestação e entre 18 e 36 anos sem distúrbios hipertensivos maternos, diabetes mellitus, vevidência de restrição de crescimento intra-uterino, suspeita de macrossomia, ruptura prematura de membranas, gravidezes múltiplas, tratamento com medicação analgésica ou antipsicótica, medicamento e dificuldades auditivas, problemas de dor crônica, dismenorréia grave, morte fetal no útero, conhecida anomalia cardiovascular ou outra anomalia fetal, ou incapacidade de compreender a escala visual analógica (VAS).

As mulheres foram divididas em grupos de controle e de intervenção e as mulheres deste último foram orientadas a escolher músicas de padrões suaves e sem mudanças bruscas na rítmica dentre músicas selecionadas de trabalhos anteriormente realizados, dentre eles temos música clássica, música clássica turca, música folclórica turca, música de arte e música popular, as sessões tinham início quando a gestante estava com dilatação a 2 cm (SMAVLI, 2014).

Ansiedade, dor, frequência cardíaca e Pressão arterial foram registrados antes dos procedimentos iniciais, na fase latente do trabalho de parto $(2-3 \mathrm{~cm}$ de dilatação), na fase ativa do trabalho de parto $(5-7 \mathrm{~cm}$ de dilatação), em dilatação completa $(10 \mathrm{~cm})$, e 2 horas após o parto (SMAVLI, 2014).

As sessões de música eram realizadas continuamente durante toda a fase latente e nas primeiras duas horas da fase ativa do trabalho de parto, com uma pequena pausa após cada hora. Após isso, o tipo de música era trocado por um que tivesse uma rítmica mais adequada ao ritmo das contrações maternas (SMAVLI, 2014).

Após avaliações, as gestantes continuaram ouvindo música até a expulsão do concepto. Uma cardiotocografia foi realizada ao fim da primeira fase de música, a escala visual analógica foi preenchida após a segunda fase e a necessidade de medicações analgésicas foi perguntada no oitavo e no vigésimo quarto dia após o parto (SMAVLI, 2014).

O estudo concluiu que houve uma diminuição da ansiedade, da dor no trabalho de parto e da utilização de analgésicos no puerpério. Houve melhora na hemodinâmica materna e nos movimentos fetais (SMAVLI, 2014).

Simavli et al (2014), em seu estudo, realizou uma análise diferentes das demais, tendo em vista que ele trabalhou a musicoterapia não em vistas de sessões, mas continuamente. Ele foi inovador também quando trabalhou a rítmica da música de acordo com o momento do trabalho de parto em que a gestante se encontrava. Além disse, o estudo se aprofundou ainda mais quando pesquisou a necessidade de analgesia no puerpério. 
Não obstante, o estudo utilizou ferramentas de avaliação tidas como ansiogênicas para a mãe, como o cardiotocógrafo. O que pode prejudicar a avaliação, subestimando o efeito da musicoterapia para a redução de ansiedade e por consequente de dor na gestante.

Por fim é possível inferir que a partir desses estudos recentes, a musicoterapia é facilmente aplicável e de grande valia para a redução de dor, ansiedade e a melhor experiência geral da mãe em relação ao parto normal.

Fica claro que os estudos precisam também delimitar melhor também neste período da gestação suas experiências com a música quanto as suas próprias características, mas os termos recorrentes usados para definir as músicas tais como: "música suave", "música calmante" ou "música clássica" podem vir a ajudar a nortear estudos futuros sobre os temas descritos.

\section{Musicoterapia no parto cesáreo}

O trabalho de parto cesáreo, embora gere menos dor no momento do nascimento quando comparado com o parto normal, é um processo doloroso que gera ansiedade medos e aflição.

Sua prevalência ainda é alta, chegando $15 \%$ no trabalho realizado por (MONGUILHOTT et al, 2018), que visava avaliar estatisticamente, como nascem os brasileiros em nosso serviço público. Nele, foi no inferido que apenas $34,8 \%$ das mães tiveram a presença de um acompanhante durante o parto, o que também é fator de sofrimento para a mãe.

Como fator isolado, o momento do pré-operatório já é um fato ansiogênico e estressante, e a música é capaz de atenuar esses fatores e melhorar o desempenho do procedimento a ser realizado. Um dos fatores que se mostram mais importantes neste processo é a escolha musical, que tem melhores resultados quando esta é feita pelo próprio paciente (COTOIA et al, 2018).

Tendo isto em vista, a musicoterapia entra como uma importante possibilidade de terapia adjuvante e de suporte para as mães que necessitam passar pelo parto cesáreo, já que a ansiedade e a dor são determinantes para a satisfação geral do processo de nascimento.

O trabalho lavrado por (KUSHNIR et al, 2012), reuniu em seu estudo uma amostra de 60 mulheres que iriam se submeter a uma cirurgia de cesárea eletiva, na qual essa possuía alguma indicação formal. Excluíram-se assim, as mulheres nas quais deram entrada no hospital e se submeteram a cesárea devido a alguma emergência obstétrica ou que não tinham nenhuma indicação para cesárea. 
Kushnir et al (2012), aplicou a escala de estado de humor, e realizou a medida frequente da frequência cardíaca, respiratória da pressão arterial sistêmica. As mulheres foram divididas em grupo experimental e grupo de controle. As mulheres eram levadas para uma ala isolada do hospital, onde ficavam separadas por cortinas, sem o contato de nenhum acompanhante, recebendo apenas visitas periódicas da equipe médica.

O grupo musical recebeu uma sessão de musicoterapia de 40 minutos, onde a música reproduzida era escolhida conforme a preferência musical da gestante dentre as seguintes possibilidades: "música popular leve", "música clássica leve” e "músicas israelenses”. Após os 40 minutos, as mulheres eram novamente submetidas aos testes novamente e por fim enviadas a sala de cirurgia (KUSHNIR et al, 2012).

Os resultados mostraram que as mulheres que eram submetidas a musicoterapia tinham uma diminuição das sensações ruins em relação ao grupo controle, ainda, as gestantes do grupo controle percebiam o procedimento como sendo menos acolhedor e mais ameaçador quando comparadas ao grupo que passou pela intervenção musical (KUSHNIR et al, 2012).

Ainda, foi possível notar que não houveram diferenças significativas nos sinais vitais das pacientes na qual foram submetidas as sessões musicais, não obstante, a pressão arterial teve significativa diminuição nessas pacientes. Isto, por si só, pode vir a demonstrar uma redução importante dos níveis de ansiedade e tensão, o que ajuda a paciente a ter uma maior sensação de satisfação. O estudo conclui que a escolha das músicas por parte das gestantes torna-se determinante devido ao fato de que isso dá uma sensação de controle para a mesma que naquele momento, encontra-se fragilizada e impotente (KUSHNIR et al, 2012).

$\mathrm{O}$ estudo, torna-se limitado quando avaliamos a amostra reduzida em comparação a outros estudos recentemente realizados. Este, usou um espaço amostral de 60 gestantes, sendo que o grupo controle, perante a escolha musical, teve que excluir duas participantes devido à baixa adesão a música clássica, o que tornava impossível avaliar seus resultados perante os demais.

O ensaio clínico conduzido por (LI; DONG, 2012) foi feito também utilizando-se de 60 participantes no espaço amostral, foram excluídas as pacientes que possuíam alguma comorbidade de coração, pulmão, fígado ou rins.

As pacientes foram divididas em grupo controle e grupo de intervenção e foram submetidas a um teste usando a escala de ansiedade de autorrevisão de zung. As pacientes eram também avaliadas utilizando-se seus sinais vitais e um holter (LI; DONG, 2012). 
As pacientes selecionavam músicas em uma playlist de música clássica chinesa e ouviam as músicas em uma posição de relaxamento por 30 minutos no dia em que seriam submetidas a cesárea. As pacientes foram submetidas aos testes e enviadas ao centro cirúrgico para a realização do procedimento. As pacientes foram orientadas quanto a necessidade de informar ao médico sempre que se sentissem desconfortáveis e preencheram a escala analógica de dor seis horas após o procedimento (LI; DONG, 2012).

$\mathrm{Na}$ análise de dados, foi possível perceber uma redução significativa da ansiedade e da dor relatada após o parto. Foi possível perceber também, uma redução da pressão arterial, frequência cardíaca e variabilidade da frequência cardíaca durante o processo de cirurgia (LI; DONG, 2012).

Tendo isso em vista, é possível inferir que o estudo realizado evidenciou uma diminuição tanto dos dados objetivos quanto dos dados subjetivos que foram pesquisados (LI; DONG, 2012). Levou-se em consideração a escolha das músicas pelas gestantes, o que é benéfico conforme discutido anteriormente, porém a escolha esteve limitada somente a um tipo de música, a "música clássica chinesa". Isso pode trazer um viés, pois limita a escolha musical da mãe, porém pode ser benéfico no que diz respeito a trazer a possibilidade de que se estude o ritmo em questão especificamente, entendendo assim seus benefícios inerentes a sua melodia aplicada a este grupo.

O ensaio clínico realizado por (SEN et al, 2010) reuniu 70 pacientes sem comorbidades, incluídas na classificação de ASA-I, entre 20 e 40 anos de idade, que passariam por uma cesárea eletiva. Estas foram divididas em grupos de controle e intervenção.

No dia anterior a cirurgia, as pacientes foram avaliadas quanto a sua escuta anterior de músicas, frequência e preferência musical. O grupo de intervenção foi submetido a uma sessão de uma hora de musicoterapia na qual não foi feita nenhuma intervenção quanto a escolha das músicas. O grupo controle foi privado de ouvir música durante o mesmo período. Após a sessão, os demais cuidados cirúrgicos, anestésicos e ventilatórios eram padronizados (SEN et al, 2010).

Foi utilizada uma escala visual analógica para avaliar a satisfação geral quanto a cirurgia e a dor na qual a paciente sentia na posição deitada e sentada. Dados objetivos também foram coletados, como a frequência cardíaca, saturação de oxigênio capilar e frequência respiratória. Quaisquer efeitos pós cirúrgicos como insônia, hipersonia, ansiedade, sono profundo, náuseas e vômitos foram citados e quantificados com um auxílio de uma escala visual analógica (SEN et al, 2010). 
Os resultados, corroboraram com outros estudos quanto aos benefícios clínicos da musicoterapia no parto cesáreo, sendo que ouve uma redução significativa da dor nas primeiras 24 horas e uma redução importante do consumo de analgésicos nas primeiras 4 horas de pósoperatório (SEN et al, 2010).

No geral os estudos aqui descritos, demostram claramente a diminuição da sensação subjetiva de dor. Há uma padronização quanto a sua pesquisa, levando em consideração que a maioria dos estudos utiliza a escala visual analógica para pesquisar isto. O mais provável é que esta seja utilizada devido à ausência de outras ferramentas que permitam a mensuração da dor.

Os estudos demonstraram também a redução objetiva do uso de medicações, seja esta mensurada nas primeiras 24 horas após o parto ou até mesmo alguns dias após. Isso nos possibilita inferir que há uma diminuição da dor tanto a longo prazo quanto a curto.

\section{Musicoterapia na obstetrícia aplicada sobre o pai}

É notável a importância da participação do pai no processo de nascimento e de pré-natal. A maioria dos pais participa do pré-natal e sente-se por muitas vezes excluído do processo e insatisfeito com a quantidade e qualidade de informações na qual recebe a respeito da gestação e do que esperar após o nascimento. Existem evidências quanto ao fato de que ações médicas no momento do pré-natal em relação aos pais podem ser eficazes para gerar uma maior participação dos pais e gerar uma maior satisfação de ambos os genitores com todo o processo de nascimento (XUE et al, 2018).

No entanto, existem poucos estudos que atendam a temática do pai durante o pré-natal, sendo o estudo publicado por (XUE et al, 2018) pioneiro neste sentido. No âmbito da musicoterapia, isso torna-se ainda mais escasso, sendo o estudo de (LABRAGUE; MCSENROE-PETIT, 2014) o único que trata desta temática até onde nós temos conhecimento.

Esse estudo é um ensaio clínico, um pré-teste prospectivo, que investigou a influência da musicoterapia em 98 pais de primeira vez, filipinos, maiores de idade que não tivessem nenhuma comorbidade evidente, principalmente doenças auditivas. Esses pais foram separados em grupo controle e grupo de intervenção musical (LABRAGUE; MCSENROE-PETIT, 2014).

As músicas selecionadas tinham característica calmante e seguiam a rítmica clássica, country e sons da natureza. As músicas tinham entre 60 e 80 batimentos por minuto, eram 
escutadas num máximo de $60 \mathrm{Db}$ e não tinham em sua harmonia instrumentos de percussão. Era oferecido aos participantes um aparelho de MP3, na qual tinha pré-gravado 500 composições na qual atendiam aos critérios anteriormente descritos. Os pacientes poderiam escolher, quais dessas músicas eles poderiam reproduzir (LABRAGUE; MCSENROE-PETIT, 2014).

Essas características estão de acordo com a maioria das bibliografias que possuem pesquisas voltadas para a mãe, sendo o batimento por minuto de acordo com a rítmica da pulsação humana, o decibilímetro da música de acordo com a média de estudos anteriores e a preferência pessoal de cada pai é levada em consideração (LABRAGUE; MCSENROE-PETIT, 2014).

A ansiedade paterna foi medida utilizando-se a escala de ansiedade linear e estado, também fortemente presentes em estudos similares. Os pais foram deslocados a uma sala a parte do hospital quando as suas respectivas conjugues estavam com $4 \mathrm{~cm}$ de dilatação. Ocorriam 20 a 40 minutos de intervenção musical. Os testes eram aplicados antes da intervenção,30 minutos após seu início e outro teste, cuja finalidade era avaliar a satisfação geral com o parto era empregada duas horas após a entrega do bebê a família (LABRAGUE; MCSENROE-PETIT, 2014).

Os estudos demonstraram que trinta minutos de intervenção musical foram eficazes para a redução da ansiedade da mesma forma que neste mesmo período, sem musicoterapia, os pais pertencentes ao grupo de controle aumentavam significativamente seus níveis de ansiedade, demonstrando que este primeiro período era especialmente importante (LABRAGUE; MCSENROE-PETIT, 2014).

No caso da satisfação geral com o parto, os pais se mostraram fortemente beneficiados neste mesmo estudo (LABRAGUE; MCSENROE-PETIT, 2014). A satisfação geral, parte do princípio do pai sentir-se apto a apoiar a mãe no processo de nascimento segundo (HANSON, 2009), conforme citado por (XAU, 2018).

Esse estudo, embora detalhado em muitos aspectos e bem embasado bibliograficamente, sugere a necessidade ainda maior de que sejam feitos mais trabalhos visando o pai durante o pré-natal (LABRAGUE; MCSENROE-PETIT, 2014). Por várias vezes, sua bibliografia cita a importância do pai neste processo, porém poucos textos são encontrados neste sentido. 


\section{A aplicação da música nos ensaios terapêuticos}

A aplicação da musicoterapia em ensaios clínicos é fundamental para a pesquisa científica no âmbito do teste de sua efetividade. No entanto, o que se observa em nosso contexto é que há uma dificuldade de uniformizar a metodologia da pesquisa científica nos trabalhos apresentados.

Em uma meta-análise realizada por (VAN WILLENSWAARD, 2017) com o intuito de avaliar os níveis de ansiedade e estresse durante a gestação foi possível observar forte discrepância entre os estilos musicais, duração das sessões de musicoterapia, diferenças entre os BPM's e volumes utilizados além de ritmos musicais.

Não existe consenso sobre a importância da preferência musical da gestante durante a terapia. Vários trabalhos utilizam musicoterapia sem distinção de como deve ser aplicada a musicoterapia ou sobre a importância de profissional treinado durante a escuta musical. Não existe uniformidade a quanto à possibilidade das sessões serem realizadas em domicílio ou em grupo em ambiente controlado.

A tabela 1, faz uma relação entre os trabalhos utilizados como bibliografia neste trabalho, o volume na qual a música é ouvida, o ritmo musical utilizado, a quantidade de batimentos por minuto da música utilizada das sessões de terapia, a quantidade e duração das sessões e os resultados apresentados.

Tabela 1 - Avaliação geral dos trabalhos de musicoterapia na obstetrícia

\begin{tabular}{|c|c|c|c|c|c|}
\hline Trabalho & Volume & Ritmo & BPM & $\begin{array}{l}\text { Quantidade } \\
\text { e duração } \\
\text { das sessões }\end{array}$ & Resultados \\
\hline ABA et al (2017) & $45 \mathrm{Db}$ & $\begin{array}{l}\text { Música } \\
\text { clássica }\end{array}$ & $\begin{array}{c}\text { Não } \\
\text { especificado }\end{array}$ & $\begin{array}{c}28 \text { minutos por } \\
\text { sessão / } 2 \\
\text { sessões }\end{array}$ & $\begin{array}{c}\text { Redução da } \\
\text { ansiedade após a } \\
\text { fertilização, } \\
\text { aumento irrisório } \\
\text { da fertilidade. }\end{array}$ \\
\hline MURPHY et al (2014) & $\begin{array}{c}\text { Não } \\
\text { especificado }\end{array}$ & $\begin{array}{l}\text { Harpa } \\
\text { melódica }\end{array}$ & $\begin{array}{c}\text { Não } \\
\text { especificado }\end{array}$ & $\begin{array}{l}20 \text { minutos por } \\
\text { sessão / } 2 \\
\text { sessões }\end{array}$ & $\begin{array}{c}\text { Redução da } \\
\text { ansiedade após a } \\
\text { fertilização, } \\
\text { discreto aumento } \\
\text { da fertilidade }\end{array}$ \\
\hline $\begin{array}{c}\text { NWEBUBE; } \\
\text { GLOVER; STEWART } \\
(2017)\end{array}$ & $\begin{array}{c}\text { Não } \\
\text { especificado }\end{array}$ & $\begin{array}{c}\text { Musica } \\
\text { calmante }\end{array}$ & $\begin{array}{c}\text { Não } \\
\text { especificado }\end{array}$ & $\begin{array}{l}20 \text { minutos } \\
\text { diários durante } \\
12 \text { semanas }\end{array}$ & $\begin{array}{c}\text { Redução dos } \\
\text { níveis de } \\
\text { ansiedade de } \\
\text { depressão }\end{array}$ \\
\hline $\begin{array}{c}\text { GONZALEZ et al } \\
\text { (2017) }\end{array}$ & $60-70 \mathrm{Db}$ & $\begin{array}{c}\text { Música } \\
\text { instrumental }\end{array}$ & 60-74 ВРМ & $\begin{array}{c}14 \text { sessões de } \\
20 \text { a } 40\end{array}$ & $\begin{array}{l}\text { Redução dos } \\
\text { níveis de }\end{array}$ \\
\hline
\end{tabular}




\begin{tabular}{|c|c|c|c|c|c|}
\hline & & & & $\begin{array}{c}\text { minutos } \\
\text { diários, três } \\
\text { vezes por } \\
\text { semana } \\
\end{array}$ & $\begin{array}{l}\text { ansiedade linear e } \\
\text { de estado }\end{array}$ \\
\hline GEBUSA et al(2017) & $65 \mathrm{Db}$ & Polca & $160-175$ & $\begin{array}{l}\text { Uma sessão de } \\
15 \text { minutos }\end{array}$ & $\begin{array}{c}\text { Aumento da } \\
\text { movimentação } \\
\text { fetal e uma } \\
\text { variabilidade da } \\
\text { frequência } \\
\text { cardíaca em 10-15 } \\
\text { BPM }\end{array}$ \\
\hline CHANG et al (2015) & $\begin{array}{c}\text { Não } \\
\text { especificado }\end{array}$ & $\begin{array}{c}\text { Crystal music, } \\
\text { música } \\
\text { clássica, sons } \\
\text { da natureza e } \\
\text { canções de } \\
\text { ninar } \\
\end{array}$ & $\begin{array}{l}\text { Variados } \\
\text { BPM's }\end{array}$ & $\begin{array}{l}14 \text { sessões } \\
\text { diárias de } 30 \\
\text { minutos }\end{array}$ & $\begin{array}{l}\text { Redução dos } \\
\text { níveis de tensão } \\
\text { sem evidencia de } \\
\text { redução dos níveis } \\
\text { gerais de estresse }\end{array}$ \\
\hline LIU et al (2015) & $\begin{array}{c}\text { Não } \\
\text { especificado }\end{array}$ & $\begin{array}{l}\text { Música } \\
\text { clássica }\end{array}$ & 60-80 BPM & $\begin{array}{l}14 \text { sessões } \\
\text { diárias de } 30 \\
\text { minutos }\end{array}$ & $\begin{array}{l}\text { Redução da } \\
\text { ansiedade, } \\
\text { depressão e } \\
\text { melhora da } \\
\text { qualidade do sono }\end{array}$ \\
\hline $\begin{array}{c}\text { TOKER; KÖMÜRCÜ } \\
\text { (2017) }\end{array}$ & $\begin{array}{c}\text { Não } \\
\text { especificado }\end{array}$ & $\begin{array}{l}\text { Música } \\
\text { clássica turca }\end{array}$ & $\begin{array}{c}\text { Não } \\
\text { especificado }\end{array}$ & $\begin{array}{l}\text { Cinco sessões } \\
\text { de } 30 \text { minutos }\end{array}$ & $\begin{array}{l}\text { Discreta redução } \\
\text { dos níveis de PA, } \\
\text { tensão e ansiedade }\end{array}$ \\
\hline $\begin{array}{c}\text { DEHCHESHMEH, } \\
\text { RAFIEI } \\
(2015)\end{array}$ & $\begin{array}{c}\text { Não } \\
\text { especificado }\end{array}$ & $\begin{array}{l}\text { Música } \\
\text { executada em } \\
\text { piano }\end{array}$ & $\begin{array}{c}\text { Não } \\
\text { especificado }\end{array}$ & $\begin{array}{l}\text { Sessão de } 30 \\
\text { minutos }\end{array}$ & $\begin{array}{l}\text { Redução da dor e } \\
\text { da ansiedade }\end{array}$ \\
\hline $\begin{array}{l}\text { TABARRO et al } \\
\text { (2010) }\end{array}$ & $\begin{array}{c}\text { Não } \\
\text { especificado }\end{array}$ & $\begin{array}{l}\text { Música } \\
\text { clássica, } \\
\text { romântica, } \\
\text { folclórica } \\
\text { japonesa }\end{array}$ & $\begin{array}{c}\text { Não } \\
\text { especificado }\end{array}$ & $\begin{array}{l}\text { Cinco sessões } \\
\text { prévias e } \\
\text { durante o } \\
\text { trabalho de } \\
\text { parto com } \\
\text { pausas } \\
\text { regulares }\end{array}$ & $\begin{array}{l}\text { Redução da dor e } \\
\text { ansiedade, } \\
\text { melhora da } \\
\text { experiência geral } \\
\text { de parto }\end{array}$ \\
\hline $\begin{array}{l}\text { SMAVLI } \\
(2014)\end{array}$ & $\begin{array}{c}\text { Não } \\
\text { especificado }\end{array}$ & $\begin{array}{l}\text { Música } \\
\text { clássica, } \\
\text { música clássica } \\
\text { turca, música } \\
\text { folclórica } \\
\text { turca, música } \\
\text { de arte e } \\
\text { música popular }\end{array}$ & $\begin{array}{c}\text { Não } \\
\text { especificado }\end{array}$ & $\begin{array}{l}\text { Durante o } \\
\text { trabalho de } \\
\text { parto com } \\
\text { pausas } \\
\text { pequenas a } \\
\text { cada hora }\end{array}$ & $\begin{array}{l}\text { Redução da dor e } \\
\text { ansiedade, } \\
\text { melhora da } \\
\text { experiência geral } \\
\text { de parto, redução } \\
\text { do uso de } \\
\text { analgesia no } \\
\text { puerpério }\end{array}$ \\
\hline KUSHNIR et al, 2012 & $\begin{array}{c}\text { Não } \\
\text { especificado }\end{array}$ & $\begin{array}{l}\text { Popular leve, } \\
\text { clássica leve e } \\
\text { musica } \\
\text { israelense }\end{array}$ & $\begin{array}{c}\text { Não } \\
\text { especificado }\end{array}$ & $\begin{array}{l}\text { Uma sessão de } \\
40 \text { minutos }\end{array}$ & $\begin{array}{c}\text { Diminuição da } \\
\text { sensação de dor e } \\
\text { diminuição do } \\
\text { medo e do } \\
\text { sentimento de } \\
\text { ameaça } \\
\text { relacionado a } \\
\text { cirurgia } \\
\end{array}$ \\
\hline $\begin{array}{l}\text { LI; DONG } \\
\quad(2012)\end{array}$ & $\begin{array}{c}\text { Não } \\
\text { especificado }\end{array}$ & $\begin{array}{l}\text { Música } \\
\text { clássica } \\
\text { chinesa }\end{array}$ & $\begin{array}{c}\text { Não } \\
\text { especificado }\end{array}$ & $\begin{array}{l}\text { Uma sessão de } \\
30 \text { minutos }\end{array}$ & $\begin{array}{c}\text { Redução da } \\
\text { ansiedade, pressão } \\
\text { arterial, frequência } \\
\text { cardíaca e } \\
\text { variabilidade da }\end{array}$ \\
\hline
\end{tabular}




\begin{tabular}{|c|c|c|c|c|c|}
\hline & & & $\begin{array}{c}\text { frequência } \\
\text { cardíaca }\end{array}$ \\
\hline $\begin{array}{c}\text { SEN et al } \\
(2010)\end{array}$ & $\begin{array}{c}\text { Não } \\
\text { especificado }\end{array}$ & Livre escolha & $\begin{array}{c}\text { Não } \\
\text { especificado }\end{array}$ & $\begin{array}{c}\text { Redução da } \\
\text { uma hora de } \\
\text { sensação de dor e } \\
\text { do uso de } \\
\text { anaugésicos }\end{array}$ \\
\hline XAU & $60 \mathrm{Db}$ & $\begin{array}{c}\text { Música } \\
\text { clássica, contry } \\
\text { e sons da } \\
\text { natureza } \\
\text { escolhidos } \\
\text { pelos pais }\end{array}$ & $\begin{array}{c}\text { Redução da } \\
\text { ansiedade paterna } \\
\text { e aumento da } \\
\text { satisfação geral do } \\
\text { pai }\end{array}$ \\
\hline
\end{tabular}

Fonte: o autor, 2018.

\section{Conclusão}

As várias fontes bibliográficas disponíveis em bases de dados demostram claramente a gama de benefícios que podem ser alcançados quando utiliza-se a musicoterapia ou mesmo a música na medicina no âmbito a obstetrícia, seja ela empregada em qualquer período da gestação ou mesmo na preconcepção ou na visão do pai.

A gama de benefícios encontrada é surpreendente, variando desde dados objetivos como redução frequência cardíaca, redução da frequência respiratória, redução nos níveis de cortisol circulante, aumento da variabilidade da frequência cardíaca fetal, aumento da motilidade fetal, diminuição do uso de medicações analgésicas no puerpério ou mesmo dados subjetivos diminuição da percepção de dor, redução dos níveis de ansiedade, redução dos níveis de estresse, redução da percepção do estado de estresse, melhora da satisfação geral do pai e da mãe quanto ao pré-natal, parto e puerpério.

No entanto, a gama de benefícios pesquisados ainda está limitada, perante a quantidade extremamente importante de benefícios que podem ser alcançados a partir da musicoterapia. Um sem número de patologias importantes ainda necessitam de investigação mais a fundo como por exemplo defeitos embrionários ou mesmo patologias corriqueiras e graves como a préeclâmpsia.

É evidente que estudos que procuram avaliar dor, ansiedade e depressão são de realização mais fácil devido a esses possuírem escalas analógicas visuais de fácil aplicação clínica, no entanto é extremamente importante que se façam estudos visando a avaliação de 
outros pontos, sendo estes levantando dados mais objetivos a fim de que seja avaliada a real potencialidade de terapias adjuvantes.

Os ensaios clínicos necessitam estudar não somente os efeitos da música no paciente, mas quais músicas causam que tipo de efeito no paciente. É sabido que existe um consenso que músicas calmas tem melhores resultados no que diz respeito a redução da dor, ansiedade e depressão, no entanto os estudos devem caminhar em conjunto, utilizando sempre uma taxa de BPM similares e uma rítmica semelhante a fim de reduzir a quantidade de vieses das pesquisas.

Existe a necessidade que se crie um padrão da quantidade de sessões que podem ser aplicadas, o tempo que cada uma deve durar entre o início e o término e o volume musical que pode ser aplicado. Façamos uma comparação com a farmacologia tradicional: sabemos que para que as terapias para que alcancem seu melhor potencial, sem causar danos, a dose do fármaco deve ser aplicada de acordo com a possibilidade de cada medicação e no horário e frequência na qual ele funcionará melhor. A musicoterapia também poderia caminhar neste sentido.

É notório que os mais diversos estudos tendem a avaliar sempre a música clássica com efeitos calmantes e um andamento lento, no entanto, isso negligencia as possibilidades dos andamentos mais agitados e seus possíveis efeitos benéficos para a gestante.

É importante também que, a partir do momento em que os estudos estejam padronizados, se façam mais estudos quanto a associação da musicoterapia em associação a outros tipos de terapias adjuvantes pois estas, tendo em vista praticamente a inexistência de efeitos colaterais, não tem malefício algum com sua aplicação conjunta.

Por fim, os estudos atuais têm mérito pois existe uma grande quantidade de trabalhos que avaliam os mais diversos períodos da gestação, desde a concepção até o puerpério. Existem dados que indicam os resultados sobre o concepto e sobre a mãe, sobre a redução da dor e sobre o contexto familiar.

Com este trabalho, ficou claro que as possibilidades da musicoterapia na obstetrícia são grandes, porém pouco conhecidas pela comunidade científica. Carece, de uma maior padronização e aprofundamento para que conheçamos ainda mais esta vertente de tratamento. Que mesmo tão antiga quanto o homem pouco se sabe sobre seu resultado na fisiologia humana. 


\section{Referências}

ABA, Yilda Arzu et al. Effect of music therapy on the anxiety levels and pregnancy rate of women undergoing in vitro fertilization-embryo transfer: A randomized controlled trial. Applied Nursing Research, [s.1.], v. 36, p.19-24, ago. 2017.

BETEGÓN, Ángela Arranz et al. A Program Aimed at Reducing Anxiety in Pregnant Women Diagnosed With a Small-for-Gestational-Age Fetus. The Journal Of Perinatal \& Neonatal Nursing, [s.1.], v. 31, n. 3, p.225-235, 2017. Ovid Technologies (Wolters Kluwer Health). http://dx.doi.org/10.1097/jpn.0000000000000270.

BAYRAMPOUR, Hamideh et al. Effect of depressive and anxiety symptoms during pregnancy on risk of obstetric interventions. Journal Of Obstetrics And Gynaecology Research, [s.1.], v. 41, n. 7, p.1040-1048, 15 mar. 2015. Wiley. http://dx.doi.org/10.1111/jog.12683.

CONRAD, Claudius. Music for healing: from magic to medicine. The Lancet V. 376 p. 11-17, 2010

COTOIA, Antonella et al. Effects of Tibetan Music on Neuroendocrine and Autonomic Functions in Patients Waiting for Surgery: A Randomized, Controlled Study. Anesthesiology Research And Practice, [s.1.], v. 2018, p.1-8, 2018. Hindawi Limited. http://dx.doi.org/10.1155/2018/9683780.

CHANDA, Mona Lisa; LEVITIN, Daniel J.. The neurochemistry of music. Trends In Cognitive Sciences, [s.1.], v. 17, n. 4, p.179-193, abr. 2013. Elsevier BV. http://dx.doi.org/10.1016/j.tics.2013.02.007

CHANG, Hsing-chi et al. The effects of music listening on psychosocial stress and maternalfetal attachment during pregnancy. Complementary Therapies In Medicine, [s.1.], v. 23, n. 4, p.509-515, ago. 2015. Elsevier BV. http://dx.doi.org/10.1016/j.ctim.2015.05.002.

DEHCHESHMEH, Faranak Safdari; RAFIEI, Hossein. Complementary and alternative therapies to relieve labor pain: A comparative study between music therapy and Hoku point ice massage. Complementary Therapies In Clinical Practice, [s.1.], v. 21, n. 4, p.229-232, nov. 2015. Elsevier BV. http://dx.doi.org/10.1016/j.ctcp.2015.09.002.

ESSAU, Cecilia A. et al. The impact of pre- and perinatal factors on psychopathology in adulthood. Journal Of Affective Disorders, [s.1.], v. 236, p.52-59, ago. 2018. Elsevier BV. http://dx.doi.org/10.1016/j.jad.2018.04.088.

GARCIA-GONZALEZ, J. et al. State-trait anxiety levels during pregnancy and foetal parameters following intervention with music therapy. Journal Of Affective Disorders, [s.1.], v. 232, p.17-22, maio 2018. Elsevier BV. http://dx.doi.org/10.1016/j.jad.2018.02.008.

GEBUZA, Grażyna et al. The effect of music therapy on the cardiac activity parameters of a fetus in a cardiotocographic examination. The Journal Of Maternal-fetal \& Neonatal Medicine, 
[s.1.], v. 30, n. 20, p.2440-2445, 22 nov. 2016. Informa UK Limited. http://dx.doi.org/10.1080/14767058.2016.1253056.

GONZÁLEZ, J. García et al. Effects of prenatal music stimulation on fetal cardiac state, newborn anthropometric measurements and vital signs of pregnant women: A randomized controlled trial. Complementary Therapies In Clinical Practice, [s.1.], v. 27, p.61-67, maio 2017. Elsevier BV. http://dx.doi.org/10.1016/j.ctcp.2017.03.004

KUSHNIR, Jonathan et al. Coping with Preoperative Anxiety in Cesarean Section: Physiological, Cognitive, and Emotional Effects of Listening to Favorite Music. Birth, [s.l.], v. 39, n. 2, p.121-127, 17 maio 2012. Wiley. http://dx.doi.org/10.1111/j.1523536x.2012.00532.x.

LABRAGUE, Leodoro J.; MCENROE-PETITTE, Denise M.. Use of Music Intervention for Reducing Anxiety and Promoting Satisfaction in First-Time Filipino Fathers. American Journal of Men's Health, [s.1.], v. 10, n. 2, p.120-127, 27 nov. 2014. SAGE Publications. http://dx.doi.org/10.1177/1557988314559240.

LI, Yang; DONG, Youjing. Preoperative music intervention for patients undergoing cesarean delivery. International Journal of Gynecology \& Obstetrics, [s.1.], v. 119, n. 1, p.81-83, 15 jul. 2012. Wiley. http://dx.doi.org/10.1016/j.ijgo.2012.05.017.

LIU, Yu-hsiang et al. Effects of music listening on stress, anxiety, and sleep quality for sleepdisturbed pregnant women. Women \& Health, [s.1.], v. 56, n. 3, p.296-311, 11 set. 2015. Informa UK Limited. http://dx.doi.org/10.1080/03630242.2015.1088116.

MONGUILHOTT, Juliana Jacques da Costa et al. Nascer no Brasil: a presença do acompanhante favorece a aplicação das boas práticas na atenção ao parto na região Sul. Revista de Saúde Pública, [s.1.], v. 52, p.1-11, 16 jan. 2018. Universidade de Sao Paulo Sistema Integrado de Bibliotecas - SIBiUSP. http://dx.doi.org/10.11606/s1518-8787.2018052006258.

MURPHY, Erin M. et al. Randomized Trial of Harp Therapy During In Vitro FertilizationEmbryo Transfer. Journal of Evidence-based Complementary \& Alternative Medicine, [s.1.], v. 19, n. 2, p.93-98, 18 dez. 2013. SAGE Publications. http://dx.doi.org/10.1177/2156587213514054.

NWEBUBE, Chineze; GLOVER, Vivette; STEWART, Lauren. Prenatal listening to songs composed for pregnancy and symptoms of anxiety and depression: a pilot study. Bmc Complementary And Alternative Medicine, [s.1.], v. 17, n. 1, p.256-261, 8 maio 2017. Springer Nature. http://dx.doi.org/10.1186/s12906-017-1759-3.

PAMIDI, Sushmita; KIMOFF, R. John. Maternal Sleep-Disordered Breathing. Chest, [s.1.], v. 153, n. 4, p.1052-1066, abr. 2018. Elsevier BV. http://dx.doi.org/10.1016/j.chest.2017.10.011.

RAGHAVAN, Rajeev; EKNOYAN, Garabed. Music, Musicians, Medicine, and the Kidney. Seminars In Dialysis, [s.1.], v. 27, n. 1, p.50-56, 30 ago. 2013. Wiley. http://dx.doi.org/10.1111/sdi.12121. 
SEN, Hüseyin et al. The efficiency and duration of the analgesic effects of musical therapy on postoperative pain. clinical trials $\mathrm{v} 22 \mathrm{n} 4 \mathrm{p}$ 145-150, 2010

SIMAVLI, Serap et al. Effect of music therapy during vaginal delivery on postpartum pain relief and mental health. Journal of Affective Disorders, [s.1.], v. 156, p.194-199, mar. 2014. Elsevier BV. http://dx.doi.org/10.1016/j.jad.2013.12.027.

TABARRO Camila Sotilo et al. Effect of the music in labor and newborn. Esc Enferm USP v. 44 p. $445-452,2010$

THAUT, Michael H.. Music as therapy in early history. Progress In Brain Research, [s.1.], p.143-158, 2015. Elsevier. http://dx.doi.org/10.1016/bs.pbr.2014.11.025.

TOKER, Eylem; KÖMÜRCÜ, Nuran. Effect of Turkish classical music on prenatal anxiety and satisfaction: A randomized controlled trial in pregnant women with preeclampsia. Complementary Therapies In Medicine, [s.1.], v. 30, p.1-9, fev. 2017. Elsevier BV. http://dx.doi.org/10.1016/j.ctim.2016.11.005.

VAN WILLENSWAARD, Kyrsten Corbijn et al. Music interventions to reduce stress and anxiety in pregnancy: a systematic review and meta-analysis. Bmc Psychiatry, [s.1.], v. 17, n. 1, p.271-280, 27 jul. 2017. Springer Nature. http://dx.doi.org/10.1186/s12888-017-1432-x.

WULFF, Verena et al. Music in Obstetrics: An Intervention Option to Reduce Tension, Pain and Stress. Geburtshilfe Und Frauenheilkunde, [s.1.], v. 77, n. 09, p.967-975, set. 2017. Georg Thieme Verlag KG. http://dx.doi.org/10.1055/s-0043-118414.

YINGER, O. S.; GOODING, L. F.. A Systematic Review of Music-Based Interventions for Procedural Support. Journal of Music Therapy, [s.1.], v. 52, n. 1, p.1-77, 1 mar. 2015. Oxford University Press (OUP). http://dx.doi.org/10.1093/jmt/thv004.

XUE, Weilin Lynn et al. Factors influencing first-time fathers' involvement in their wives' pregnancy and childbirth: A correlational study. Midwifery, [s.1.], v. 62, p.20-28, jul. 2018. Elsevier BV. http://dx.doi.org/10.1016/j.midw.2018.03.002.

\section{Como citar este artigo (Formato ABNT):}

MESQUITA NETO, Edmar; MELO, Antonio Marlos D. de; NEVES, Ana Beatriz C. S.; NEVES, Isabela Callou S.; SAMPAIO, José Angelo A.; PETRÔNIO, Cícera Nerilda V.; TELLES, Maria Valéria Leimig ; MALHEIRO, Djailson Ricardo. A Musicoterapia e a Música na Medicina como terapia adjuvante no âmbito da Obstetrícia: Uma Revisão Bibliográfica. Id on Line Revista Multidisciplinar e de Psicologia, 2018, vol.12, n.40, p.1139-1165. ISSN: 1981-1179.

Recebido: 28.05.2018

Aceito: 29.05.2018 